\title{
CENTRAL LIMIT THEOREMS FOR LAW-INVARIANT COHERENT RISK MEASURES
}

\author{
DENIS BELOMESTNY*** AND \\ VOLKER KRÄTSCHMER, ${ }^{* * * *}$ University of Duisburg-Essen
}

\begin{abstract}
In this paper we study the asymptotic properties of the canonical plugin estimates for law-invariant coherent risk measures. Under rather mild conditions not relying on the explicit representation of the risk measure under consideration, we first prove a central limit theorem for independent and identically distributed data, and then extend it to the case of weakly dependent data. Finally, a number of illustrating examples is presented.
\end{abstract}

Keywords: Law-invariant coherent risk measure; canonical plugin estimate; functional central limit theorem; weak dependence

2010 Mathematics Subject Classification: Primary 60F05; 62F12

Secondary $60 \mathrm{~F} 17 ; 62 \mathrm{G} 30 ; 91 \mathrm{~B} 30$

\section{Introduction}

In the seminal paper [1] the authors introduced the concept of coherent risk measures as a mathematical tool to assess the risks of financial positions. Formally, these objects are functionals on sets of random variables expressing risks of financial positions. The functionals should fulfill some defining properties which are axiomatic in nature to give a foundation for a normative risk assessment from the viewpoint of a regulator. An alternative axiomatic approach from the perspective of financial investors has been provided in [11], leading to the more general notion of convex risk measures.

During the last decade coherent risk measures identifying risks of financial positions with identical distributions, the so-called law-invariant coherent risk measures, have become popular in some applied fields. They are building blocks in quantitative risk management (see [20]), and they have been suggested as a systematic approach for calculations of insurance premia (cf. [14]). Moreover, viewed as statistical functionals on sets of distribution functions, they satisfy the property of being monotone with respect to the second-order stochastic dominance (cf. [3]; for general information on stochastic orders, see [23]). This illustrates the genuine intuition of risk measures as indices of distributions, emphasizing the downsize risk of underlying financial positions.

In practice, we often face the problem of estimating the values of law-invariant coherent risk measures from a time series. A customary approach is to replace the unknown distribution function with its empirical counterpart based on observed data and then to plug this estimate into the risk measure to obtain its estimate. In this paper we study the asymptotic properties of the resulting plugin estimates. Such an asymptotic analysis might be, for example, helpful for

Received 28 September 2010; revision received 10 October 2011.

* Postal address: Faculty of Mathematics, University of Duisburg-Essen, D-47057 Duisburg, Germany.

Research supported by the Deutsche Forschungsgemeinschaft through SFB 649 'Economic Risk'.

** Email address: denis.belomestny@uni-due.de

*** Email address: volker.kraetschmer@uni-due.de 
constructing confidence sets or performing statistical tests. Asymptotic properties of the plugin estimates for coherent risk measures have been investigated in two recent works, namely in [6] and [25]. While Pflug and Wozabal [25] provided general results for a class of coherent risk measures in the case of independent data, Beutner and Zähle [6] used a new functional delta method to obtain limit distributions for the subclass of concave distortion risk measures in the case of strongly mixing data.

In both the abovementioned articles the results are based on general methods which do not take into account specific properties of the law-invariant coherent risk measures, leading to unnecessarily strong assumptions on the underlying distribution. The aim of this paper is to extend and systemize the results on central limit theorems (CLTs) for plugin estimates of lawinvariant coherent risk measures. The contribution of the paper is twofold. On the one hand, we prove CLTs for plugin estimates for a rather general class of coherent risk measures under less restrictive assumptions, taking into account the fact that the 'loss' tails are more relevant than the 'gain' tails for coherent risk measures. On the other hand, in contrast to the previous literature our results do not rely on the knowledge of the specific representations for the risk measures, expressing the assumptions just in terms of the functionals themselves. Last but not least, we extend our CLT to case-dependent observations and discontinuous distributions.

The paper is organized as follows. After introducing the main setup in Section 2 we present our main results in Section 3 for independent data. These results will then be extended to the case of dependent data in Section 4. Section 5 gathers some auxiliary results to prove the main results, whereas in Section 6 we give their proofs. Section 7 is devoted to the proofs of the main results. Some useful technical results will be formulated and shown in the appendices.

\section{Main setup}

Let $\mathbb{F} x$ be a set of distribution functions on $\mathbb{R}$ related to a vector space $\mathcal{X}$ of integrable random variables on some atomless probability space $(\Omega, \mathcal{F}, \mathrm{P})$ enclosing all P-essentially bounded spaces. A mapping $\rho: \mathbb{F}_{X} \rightarrow \mathbb{R}$ is called a law-invariant coherent risk measure if the following conditions are fulfilled.

Monotonicity. For any $X_{1}, X_{2} \in \mathcal{X}$ with $F_{X_{1}}(x) \leq F_{X_{2}}(x), x \in \mathbb{R}$,

$$
\rho\left(F_{X_{1}}\right) \leq \rho\left(F_{X_{2}}\right) .
$$

Cash invariance. For any $X \in \mathcal{X}$ and $c \in \mathbb{R}$,

$$
\rho\left(F_{X+c}\right)=\rho\left(F_{X}\right)-c .
$$

Sublinearity. For any $X_{1}, X_{2} \in \mathcal{X}$ and $\lambda_{1}, \lambda_{2} \geq 0$,

$$
\rho\left(F_{\lambda_{1} X_{1}+\lambda_{2} X_{2}}\right) \leq \lambda_{1} \rho\left(F_{X_{1}}\right)+\lambda_{2} \rho\left(F_{X_{2}}\right) .
$$

Here $F_{Z}$ stands for the distribution function of the random variable $Z$. The defining properties of the coherent risk measures correspond to their well-known interpretations as representing risk attitudes of financial investors (cf. [11, Chapter 4]). Let $\left(X_{i}\right)_{i \in \mathbb{N}}$ be an independent sequence of real random variables defined on $(\Omega, \mathcal{F}, \mathrm{P})$ with common distribution function $F$ and related left-continuous quantile function $q_{F}$. Additionally, define, $q_{F}(0):=q_{F}(0+)$ as well as $q_{F}(1):=q_{F}(1-)$. Denote by $F_{n}$ the empirical distribution function based on the sample $\left(X_{1}, \ldots, X_{n}\right)$ and set $\rho_{n}(F):=\rho\left(F_{n}\right)$. The main goal of this paper is to study the 
asymptotic properties of the process $\left(\sqrt{n}\left(\rho_{n}(F)-\rho(F)\right)\right)_{n \in \mathbb{N}}$. As an important tool, let us consider the mapping

$$
\psi_{\rho}:[0,1] \rightarrow[0,1], \quad t \mapsto \rho\left(F_{-B(1, t)}\right),
$$

where $B(1, t)$ stands for Bernoulli random variable with expectation $t$. This mapping is a distortion function, i.e. it is nondecreasing with $\psi_{\rho}(0)=0$ and $\psi_{\rho}(1)=1$, suggesting the name associated distortion function.

\section{Main results}

In order to prove a CLT for the process $\left(\sqrt{n}\left(\rho_{n}(F)-\rho(F)\right)\right)_{n \in \mathbb{N}}$, we need the following two assumptions.

(AC) $\mathcal{X}$ is a Stonean vector lattice, i.e. here $X \wedge Y, X \vee Y \in \mathcal{X}$ for $X, Y \in \mathcal{X}$, and $\rho$ satisfies

$$
\lim _{k \rightarrow \infty} \rho\left(F_{-(X-k)^{+}}\right)=0 \quad \text { for nonnegative } X \in X, \quad \lim _{t \rightarrow 0+} \psi_{\rho}(t)=0 .
$$

(AI) The stationary distribution function $F$ of the sequence $\left(X_{i}\right)_{i \in \mathbb{N}}$ fulfills the following integrability condition:

$$
\int_{\mathbb{R}} F(x)^{-1 / 2}(1-F(x))^{1 / 2} \psi_{\rho}(\lambda F(x)) \mathrm{d} x<\infty
$$

for some $\lambda \in\left(0, \frac{1}{2}\right)$.

The main result of our study is the following theorem, which gives the asymptotic distribution of the process $\left(\sqrt{n}\left(\rho_{n}(F)-\rho(F)\right)\right)_{n \in \mathbb{N}}$.

Theorem 3.1. Let $F$ have a finite set $D(F)$ of discontinuity points such that the restriction of $F$ to $\left(q_{F}(0), q_{F}(1)\right) \backslash D(F)$ is continuously differentiable with strictly positive derivative.

Then, under assumptions $(A C)$ and $(A I)$, we can find a set $S(\rho(F))$ of continuous, concave distortion functions which is compact with respect to the uniform metric, and there exists some centered Gaussian process $\left(G_{\psi}\right)_{\psi \in S(\rho(F))}$ with continuous paths and

$$
\mathrm{E}\left[G\left(\psi_{1}\right) G\left(\psi_{2}\right)\right]=\int_{\mathbb{R}^{2}} \psi_{1}^{\prime}(F(x)) \psi_{2}^{\prime}(F(y))[F(x \wedge y)-F(x) F(y)] \mathrm{d} x \mathrm{~d} y
$$

for any $\psi_{1}, \psi_{2} \in S(\rho(F))$ such that the sequence $\left(\sqrt{n}\left[\rho_{n}(F)-\rho(F)\right]\right)_{n \in \mathbb{N}}$ converges in law to $\max _{\psi \in S(\rho(F))} G_{\psi}$. Here $\psi^{\prime}$ denotes the right-sided derivative of $\psi$. Moreover, if

$$
\mathrm{E}\left[G\left(\psi_{1}\right)-G\left(\psi_{2}\right)\right]^{2} \neq 0
$$

for any two different $\psi_{1}, \psi_{2} \in S(\rho(F))$ then $\sup _{\psi \in S(\rho(F))} G(\psi)=G(Z)$ for some Borel random element $Z$ of $S(\rho(F))$.

Proof. See Section 7.

Remark 3.1. As it will become clear from the proof of Theorem 3.1, $S(\rho(F))$ consists of continuous concave distortion functions $\psi$ satisfying

$$
\rho(F)=\int_{-\infty}^{0} \psi(F(x)) \mathrm{d} x-\int_{0}^{\infty}[1-\psi(F(x))] \mathrm{d} x .
$$

In particular, $\psi \leq \psi_{\rho}$ for any $\psi \in S(\rho(F))$. 
Remark 3.2. Assumption (AC) is always fulfilled if there is some topologically complete seminorm $\|\cdot\|$ on the Stonean vector lattice $\mathcal{X}$ such that the following properties are satisfied:

$$
\begin{gathered}
\|X\| \leq\|Y\| \quad \text { for }|X| \leq|Y|, \mathrm{P} \text {-almost surely (P-a.s.), } \\
\lim _{k \rightarrow \infty}\left\|X_{k}\right\|=0 \quad \text { whenever } X_{k} \nearrow 0, \mathrm{P} \text {-a.s. }
\end{gathered}
$$

(cf. [30]). General classes of random variables meeting these requirements are given by

$$
\mathcal{M}^{g}(\Omega, \mathcal{F}, \mathrm{P}):=\left\{Y \in \mathcal{L}^{0}(\Omega, \mathcal{F}, \mathrm{P}) \mid \mathrm{E}\left[g\left(\frac{|Y|}{c}\right)\right]<\infty \text { for all } c>0\right\},
$$

where $g$ denotes any continuous Young function, i.e. a continuous, nondecreasing, unbounded, convex function $g: \mathbb{R}_{+} \rightarrow \mathbb{R}_{+}$with $g(0)=0$. These classes may be equipped with the Luxemburg seminorm $\|\cdot\|_{g}$ defined by

$$
\|Y\|_{g}:=\inf \left\{c>0 \mid \mathrm{E}\left[g\left(\frac{|Y|}{c}\right)\right] \leq 1\right\},
$$

being complete, and satisfying conditions (3.1) and (3.2) (see [9, Theorems 2.1.11, 2.1.14]).

Let us turn now to some examples.

Example 3.1. An important class of law-invariant coherent risk measures consists of the so-called concave distortion risk measures. To recall, the concave distortion risk measure $\rho=: \rho_{\psi}$ with respect to a concave distortion function $\psi$ is defined by

$$
\rho_{\psi}\left(F_{X}\right)=\int_{-\infty}^{0} \psi\left(F_{X}(x)\right) \mathrm{d} x-\int_{0}^{\infty}\left[1-\psi\left(F_{X}(x)\right)\right] \mathrm{d} x
$$

(cf., e.g. [8] or [11]). Note that $\psi_{\rho_{\psi}}=\psi$ holds.

The risk measure can be viewed as a Choquet integral with respect to the set function $\psi(\mathrm{P}(\cdot))$ (cf. [8]), and $\mathbb{F} x$ consists of all distribution functions on $\mathbb{R}$ such that each integral in representation (3.3) is finite. The set $\mathcal{X}$ of random variables on $(\Omega, \mathcal{F}, \mathrm{P})$ whose distribution functions belong to $\mathbb{F}_{X}$ is indeed a linear space satisfying $X \wedge Y, X \vee Y \in \mathcal{X}$ for $X, Y \in \mathcal{X}$ (cf. [8, Proposition 9.5 with Proposition 9.3]). If, in addition, $\psi$ is continuous then

$$
\|X\|_{\psi}:=\int_{0}^{\infty} \psi\left(1-F_{|X|}(x)\right) \mathrm{d} x
$$

defines a topologically complete seminorm on $\mathcal{X}$ satisfying conditions (3.1) and (3.2) (cf. [8, Theorems 8.9 and 9.5]).

The choice $\psi(u)=1 / \alpha(u \wedge \alpha)$ with $\alpha \in(0,1]$ leads to

$$
\rho_{\psi}\left(F_{X}\right):=\int \mathbf{1}_{(0, \alpha]}(\beta) q_{X}(\beta) \mathrm{d} \beta=\mathrm{AV} @ \mathrm{R}_{\alpha}(X),
$$

where $q_{X}$ denotes any quantile function of the distribution function $F_{X}$ of $X$. It is known as the average value at risk at level $\alpha$, and it is well defined for $\mathcal{X}=\mathcal{L}^{1}(\Omega, \mathcal{F}, \mathrm{P})$.

If $\psi_{\rho}$ is continuous, and if $F$ is as in Theorem 3.1, then it follows from the application of Theorem 3.1 along with Remark 3.1 that, under assumption (AI), the sequence $\left(\sqrt{n}\left[\rho_{n}(F)-\right.\right.$ $\rho(F)])_{n \in \mathbb{N}}$ converges in law to a centered normally distributed random variable with variance $\sigma^{2}$ satisfying

$$
\sigma^{2}=\int_{\mathbb{R}^{2}} \psi_{\rho}^{\prime}(F(x)) \psi_{\rho}^{\prime}(F(y))[F(x \wedge y)-F(x) F(y)] \mathrm{d} x \mathrm{~d} y .
$$


Example 3.2. Setting

$$
\rho(X)=-\mathrm{E}[X]+a\left\|(X-\mathrm{E}[X])^{-}\right\|_{p}, \quad a \in[0,1], p \in[1, \infty),
$$

for all $X \in L^{p}(\Omega, \mathcal{F}, \mathrm{P})$ we arrive at the so-called one-sided moment coherent risk measure (see [10]). The associated distortion function $\psi_{\rho}$ satisfies $\psi_{\rho}(t)=t+a(1-t) t^{1 / p}$. Hence, assumption $(\mathrm{AI})$ reads

$$
\int_{\mathbb{R}}[F(x)(1-F(x))]^{1 / 2}\left[1+a(1-\lambda F(x))(\lambda F(x))^{1 / p-1}\right] \mathrm{d} x<\infty \quad \text { for some } \lambda \in\left(0, \frac{1}{2}\right),
$$

which is always fulfilled in the case when

$$
\int_{\mathbb{R}}[F(x)(1-F(x))]^{1 / 2} F(x)^{1 / p-1} \mathrm{~d} x<\infty .
$$

Example 3.3. Let $g$ be a strictly increasing continuous Young function satisfying $g(1)=1$, and let $\mathcal{X}$ be the space $\mathcal{M}^{g}(\Omega, \mathcal{F}, \mathrm{P})$ associated with $g$ as in Remark 3.2. Moreover, fix $\alpha \in(0,1)$. It was shown in [12] that, for every $X \in \mathcal{M}^{g}(\Omega, \mathcal{F}, \mathrm{P})$ and every $x \in \mathbb{R}$ with $1-F_{X}(x)>0$, there exists a unique real number $\pi_{\alpha}^{g}(X, x)>x$ such that

$$
\mathrm{E}\left[g\left(\frac{(X-x)^{+}}{\pi_{\alpha}^{g}(X, x)-x}\right)\right]=1-\alpha .
$$

Therefore, we can define a functional $\rho_{\alpha}^{H, g}$ on the set $\mathbb{F}^{g}$ of all distribution functions $F_{X}$ of random variables $X$ from $\mathcal{M}^{g}(\Omega, \mathcal{F}, \mathrm{P})$ by

$$
\rho_{\alpha}^{H, g}\left(F_{X}\right):=\inf \left\{\pi_{\alpha}^{g}(-X, x) \mid x \in \mathbb{R} \text { with } 1-F_{-X}(x)>0\right\} .
$$

Indeed, $\rho_{\alpha}^{H, g}$ is a law-invariant coherent risk measure (see [4] and [16]) which satisfies assumption (AC) in view of Remark 3.2. Moreover, it is easy to check that we have, for $t \in(0,1)$,

$$
\psi_{\rho_{\alpha}^{H, g}}(t) \leq 1 \wedge\left(t+\frac{1-t}{g^{-1}((1-\alpha) / t)}\right)=: \hat{\psi}_{\rho_{\alpha}^{H, g}}(t)
$$

where $g^{-1}$ denotes the inverse of $g$ (recall that we assumed that the Young function $g$ was strictly increasing). Hence, we may replace $\psi_{\rho_{\alpha}^{H}, g}$ with $\hat{\psi}_{\rho_{\alpha}^{H, g}}^{H}$ when verifying assumption (AI).

Recently, Müller pointed out that expectiles, originally introduced in [24], may be viewed as law-invariant coherent risk measures (cf. [22]).

Example 3.4. The expectiles-based risk measure with respect to any fixed $\alpha \in\left[\frac{1}{2}, 1\right)$ is defined by

$$
\rho\left(F_{X}\right)=\underset{x \in \mathbb{R}}{\arg \min }\left[(1-\alpha)\left\|((-X)-x)^{-}\right\|_{2}^{2}+\alpha\left\|((-X)-x)^{+}\right\|_{2}^{2}\right]
$$

for all $X \in \mathcal{L}^{2}(\Omega, \mathcal{F}, \mathrm{P})$. The associated distortion function $\psi_{\rho}$ satisfies

$$
\psi_{\rho}(t)=\frac{\alpha t}{1-\alpha+t(2 \alpha-1)} .
$$

In particular, assumption (AI) is equivalent to

$$
\int_{\mathbb{R}} \frac{\sqrt{F(x)(1-F(x))}}{1-\alpha+\lambda F(x)(2 \alpha-1)} \mathrm{d} x<\infty \quad \text { for some } \lambda \in\left(0, \frac{1}{2}\right) .
$$


Discussion. Pflug and Wozabal [25] studied CLTs for the distortion risk measures discussed in Example 3.1. Motivated by earlier results on limit theorems for $L$-statistics they implicitly assumed that $\sup _{t \in(0,1)} \psi_{\rho}(t) / t^{\beta}<\infty$ for some $\beta \in\left(0, \frac{1}{2}\right]$ and

$$
\left|q_{F}(t)\right| \leq C[t(1-t)]^{-d}, \quad t \in(0,1),
$$

for some $d \in\left(-\infty, \beta-\frac{1}{2}\right)$. First, note that, in contrast to (3.4), our assumption (AI) concerns only the left tail of the distribution $F$. Furthermore, the next example shows that the tail condition (3.4) is substantially more restrictive than assumption (AI). Define $\psi(t):=\sqrt{t}[1+$ $\ln (100)] /[1+\ln (100)-\ln (t)]$ to be a concave distortion function which induces a concave distortion risk measure $\rho_{\psi}$, say, as in Example 3.1. It is obvious that in this case the tail condition (3.4) is satisfied for distributions with lower-bounded support only, in contrast to assumption (AI). Indeed, for $\rho_{\psi}$, assumption (AI) reads

$$
\int_{q_{F}(0)}^{q_{F}(1)} \frac{\sqrt{1-F(x)}}{1+\ln (100)-\ln (F(x))} \mathrm{d} x<\infty .
$$

Invoking the well-known expansions for the Gaussian error function, it may be seen that the above condition is satisfied for any normal distribution $F$.

\section{Extension to dependent data}

In this section we carry over the results of the previous section to the case of dependent observations $X_{1}, \ldots, X_{n}$. First, let us impose the following mixing assumption.

(AM) The sequence $\left(X_{i}\right)_{i \in \mathbb{N}}$ is strictly stationary and strongly mixing with mixing coefficients $\alpha(i)$ satisfying

$$
\alpha(i) \leq \bar{\alpha}_{0} \exp \left(-\bar{\alpha}_{1} i\right), \quad i \in \mathbb{N},
$$

for some constants $\bar{\alpha}_{0}>0$ and $\bar{\alpha}_{1}>0$.

Remark 4.1. As an example of stationary sequences fulfilling the mixing assumption (AM), we may take ARMA processes with continuously distributed innovations (cf. [21]) or GARCH processes with continuously distributed innovations and Lebesgue density being positive in a neighborhood of 0 (cf. [18]). For further examples and general conditions, see [19].

In order to extend Theorem 3.1 to dependent data, we also have to modify assumption (AI) and replace it by the following assumption.

(AI') The common distribution function $F$ of the sequence $\left(X_{i}\right)_{i \in \mathbb{N}}$ fulfills the following integrability condition:

$$
\int_{q_{F}(0)}^{q_{F}(1)} F(x)^{-1 / 2-2 \delta}(1-F(x))^{1 / 2-\delta} \psi_{\rho}\left(\lambda F(x)^{1+\delta}\right) \mathrm{d} x<\infty
$$

for some $\delta, \lambda \in\left(0, \frac{1}{2}\right)$.

We are now ready to formulate the main result of this section concerning the asymptotic distribution of $\sqrt{n}\left(\rho_{n}(F)-\rho(F)\right)$.

Theorem 4.1. Let $F$ have a finite set $D(F)$ of discontinuity points such that the restriction of $F$ to $\left(q_{F}(0), q_{F}(1)\right) \backslash D(F)$ is continuously differentiable with strictly positive derivative. Then, under assumptions (AC), (AI'), and (AM), we may find a set $S(\rho(F))$ of continuous, concave distortion functions which is compact with respect to the uniform metric, and there exists some 
centered Gaussian process $\left(G_{\psi}\right)_{\psi \in S(\rho(F))}$ with continuous paths and

$$
\begin{aligned}
& \mathrm{E}\left[G\left(\psi_{1}\right) G\left(\psi_{2}\right)\right] \\
& =\int_{\mathbb{R}^{2}} \psi_{1}^{\prime}(F(x)) \psi_{2}^{\prime}(F(y)) \\
& \quad \times\left[F(x \wedge y)-F(x) F(y)+2 \sum_{k=1}^{\infty}\left(\mathrm{P}\left(X_{1} \leq x, X_{k} \leq y\right)-F(x) F(y)\right)\right] \mathrm{d} x \mathrm{~d} y
\end{aligned}
$$

for any $\psi_{1}, \psi_{2} \in S(\rho(F))$ such that the sequence $\left(\sqrt{n}\left[\rho_{n}(F)-\rho(F)\right]\right)_{n \in \mathbb{N}}$ converges in law to $\max _{\psi \in S(\rho(F))} G(\psi)$. Moreover, if

$$
\mathrm{E}\left[G\left(\psi_{1}\right)-G\left(\psi_{2}\right)\right]^{2} \neq 0
$$

for any two different $\psi_{1}, \psi_{2} \in S(\rho(F))$ then $\sup _{\psi \in S(\rho(F))} G(\psi)=G(Z)$ for some Borel random element $Z$ of $S(\rho(F))$.

Proof. See Section 7.

\section{Auxiliary results}

In this section we formulate some auxiliary results needed to prove Theorems 3.1 and 4.1.

Proposition 5.1. Under assumption (AC), there exists a set $\Psi$ of continuous, concave distortion functions which is compact with respect to the uniform metric on $[0,1]^{[0,1]}$ such that

$$
\rho=\sup _{\psi \in \Psi} \rho_{\psi}
$$

Proof. See Appendix B.

According to Proposition 5.1 we can restrict considerations to the risk measure $\rho$ admitting representation $\rho=\sup _{\psi \in \Psi} \rho_{\psi}$ for some set $\Psi$ of continuous, concave distortion functions which is compact with respect to the uniform metric on $[0,1]^{[0,1]}$. Then we can write $\sqrt{n}\left[\rho_{n}(F)-\right.$ $\rho(F)]=\sqrt{n}\left[\sup _{\psi \in \Psi} \rho_{\psi}\left(F_{n}\right)-\sup _{\psi \in \Psi} \rho_{\psi}(F)\right]$. Let us now consider the auxiliary stochastic processes $\left(\mathscr{D}_{n}(\psi)\right)_{\psi \in \Psi}(n \in \mathbb{N})$, where

$$
\mathscr{D}_{n}(\psi):=\sqrt{n}\left[\rho_{\psi}\left(F_{n}\right)-\rho_{\psi}(F)\right]=\sqrt{n} \int_{\mathbb{R}}\left[\psi\left(F_{n}(x)\right)-\psi(F(x))\right] \mathrm{d} x, \quad \psi \in \Psi .
$$

They have paths in the space $l^{\infty}(\Psi)$ defined to consist of all bounded, real-valued mappings on $\Psi$. Endowing $l^{\infty}(\Psi)$ with the uniform topology, we will show next that the mapping $\mathcal{D}_{n}: \Psi \mapsto \mathbb{R}^{\Omega}$ can be viewed as a Borel random element of $l^{\infty}(\Psi)$. The idea is to reduce the proofs of Theorems 3.1 and 4.1 to a convergence in law of the sequence of $\left(\mathscr{D}_{n}\right)_{n}$ in $l^{\infty}(\Psi)$. This would allow us to apply the functional delta method for sup functionals to obtain the desired convergence results for $\left(\sqrt{n}\left[\rho_{n}(F)-\rho(F)\right]\right)_{n}$ (see [28]).

First, we have

$$
|\psi(t)-\psi(s)| \leq \psi_{\rho}(|t-s|) \text { for } t, s \in[0,1]
$$

(cf. [16]). Moreover, observe that concavity of each $\psi \in \Psi$ implies that

$$
\begin{aligned}
|\psi(t)-\psi(s)| & =\left|\int_{s}^{t} \psi^{\prime}(u) \mathrm{d} u\right| \\
& \leq\left|\psi^{\prime}(s)\right||s-t|
\end{aligned}
$$




$$
\begin{aligned}
& \leq|s-t| \frac{\psi(s)-\psi(\gamma s)}{(1-\gamma) s} \\
& \leq|s-t| \frac{\psi_{\rho}((1-\gamma) s)}{(1-\gamma) s}
\end{aligned}
$$

holds for $s, \gamma \in(0,1)$ and $t \in[s, 1]$, where henceforth $\psi^{\prime}$ denotes the right-sided derivative of $\psi$. The following technical auxiliary result will turn out to be useful later on.

Lemma 5.1. If either (AI) or (AI') is satisfied, then the set

$$
\left\{\psi(F) \mathbf{1}_{(-\infty, 0]}-[1-\psi(F)] \mathbf{1}_{(0, \infty)} \mid \psi \in \Psi\right\}
$$

is dominated by a mapping which is integrable with respect to the ordinary Lebesgue-Borel measure on $\mathbb{R}$.

Proof. We will only show the statement of Lemma 5.1 under assumption (AI'); the respective proof under assumption (AI) follows the same line of reasoning.

Let $\delta, \lambda \in\left(0, \frac{1}{2}\right]$, as in (AI'). By the concavity of $\psi$ we have

$$
\begin{aligned}
F(x)^{\delta} \psi(F(x)) & \leq \frac{1}{\lambda} \psi\left(\lambda F(x)^{1+\delta}\right) \\
& \leq \frac{1}{\lambda} F(x)^{-1 / 2-\delta}(1-F(x))^{1 / 2-\delta} \psi\left(\lambda F(x)^{1+\delta}\right) F(x)^{1 / 2+\delta}(1-F(x))^{\delta-1 / 2} .
\end{aligned}
$$

Hence, in view of (5.1) we obtain, for $x<q_{F}\left(\frac{1}{2}\right)$,

$$
\psi(F(x)) \leq \frac{2}{\lambda} F(x)^{-1 / 2-2 \delta}(1-F(x))^{1 / 2-\delta} \psi_{\rho}\left(\lambda F(x)^{1+\delta}\right) .
$$

Furthermore, by (5.2) and the concavity of $\psi$,

$$
\begin{aligned}
1-\psi(F(x)) & =\int_{F(x)}^{1} \psi^{\prime}(u) \mathrm{d} u \\
& \leq(1-F(x)) \psi^{\prime}(F(x)) \\
& \leq(1-F(x)) \psi^{\prime}\left(\lambda F(x)^{1+\delta}\right) \\
& \stackrel{(5.2)}{\leq} 2 \frac{\psi_{\rho}\left(\lambda F(x)^{1+\delta} / 2\right)}{\lambda F(x)^{1+\delta}}(1-F(x)) \\
& \leq 2 \frac{\psi_{\rho}\left(\lambda F(x)^{1+\delta}\right)}{\lambda F(x)^{1+\delta}}(1-F(x))
\end{aligned}
$$

for $F(x)>0$. This implies that, for $x>q_{F}\left(\frac{1}{2}\right)$,

$$
1-\psi(F(x)) \leq \frac{2}{\lambda} F(x)^{-1 / 2-2 \delta}(1-F(x))^{1 / 2-\delta} \psi_{\rho}\left(\lambda F(x)^{1+\delta}\right) .
$$

Since $\left\{\psi(F) \mathbf{1}_{(-\infty, 0]}-[1-\psi(F)] \mathbf{1}_{(0, \infty)} \mid \psi \in \Psi\right\}$ is uniformly bounded, we may conclude the statement of Lemma 5.1 from (5.3), (5.4), and assumption (AI').

As a first consequence of Lemma 5.1, we can show that within our setting the paths of the processes $\left(\mathscr{D}_{n}(\psi)\right)_{\psi \in \Psi}$ are uniformly continuous.

Lemma 5.2. If either (AI) or (AI') is satisfied, then each process $\left(\mathscr{D}_{n}(\psi)\right)_{\psi \in \Psi}$ has uniformly continuous paths with respect to the uniform metric. 
Proof. Since $\Psi$ is compact, the paths of any process $\left(\mathscr{D}_{n}(\psi)\right)_{\psi \in \Psi}$ are uniformly continuous if and only if they are continuous. So it suffices to show the continuity of the paths.

Let $\left(\psi_{k}\right)_{k}$ denote any sequence in $\Psi$ which converges to some $\psi \in \Psi$ with respect to the uniform metric. Denoting the sample minimum and maximum of $\left(X_{1}, \ldots, X_{n}\right)$ by $X_{n: 1}$ and $X_{n: n}$, respectively, we observe that

$$
\left|\psi_{k}\left(F_{n}\right) \mathbf{1}_{(-\infty, 0]}-\left[1-\psi_{k}\left(F_{n}\right)\right] \mathbf{1}_{(0, \infty)}\right| \leq \mathbf{1}_{\left[X_{n: 1} \wedge 0, X_{n: n} \vee 0\right]} .
$$

Hence, in view of Lemma 5.1, $\left\{\psi_{k}\left(F_{n}\right)-\psi_{k}(F) \mid k \in \mathbb{N}\right\}$ is P-a.s. dominated by mappings which are integrable with respect to the ordinary Lebesgue-Borel measure $\lambda^{1}$ on $\mathbb{R}$. This completes the proof due to the dominated convergence theorem.

The uniform metric on $\Psi$ is separable due to compactness, so, by Lemma 5.2, the mappings $\mathcal{D}_{n}$ are Borel random elements of $\mathrm{UCB}(\Psi)$, the space of bounded real-valued mappings on $\Psi$ which are uniformly continuous with respect to the supremum metric, where $\operatorname{UCB}(\Psi)$ is equipped with the supremum norm $\|\cdot\|_{\infty}$. Hence, the map $\mathscr{D}_{n}: \Psi \mapsto \mathbb{R}$ can be viewed as a Borel random element of $l^{\infty}(\Psi)$.

We will show the following result concerning the convergence of $\left(\mathscr{D}_{n}\right)_{n}$.

Theorem 5.1. Let the assumptions of either Theorem 3.1 or Theorem 4.1 be fulfilled. Then there exists a tight centered Gaussian Borel random element $G$ of $\mathrm{UCB}(\Psi)$ with

$$
\begin{aligned}
& \mathrm{E}\left[G\left(\psi_{1}\right) G\left(\psi_{2}\right)\right] \\
& =\int_{\mathbb{R}^{2}} \psi_{1}^{\prime}(F(x)) \psi_{2}^{\prime}(F(y)) \\
& \quad \times\left[F(x \wedge y)-F(x) F(y)+2 \sum_{k=1}^{\infty}\left(\mathrm{P}\left(X_{1} \leq x, X_{k} \leq y\right)-F(x) F(y)\right)\right] \mathrm{d} x \mathrm{~d} y
\end{aligned}
$$

for any $\psi_{1}, \psi_{2} \in \Psi$ such that $\left(\mathscr{D}_{n}(\psi)\right)_{\psi \in \Psi}$ converges in law to $G$.

For the proof of Theorem 5.1 we will verify the following two results whose formulations need some preparation. By assumption on $F$ we can find $q_{F}(0)=: a_{0}<a_{1}<\cdots<a_{r+1}:=$ $q_{F}(1)$ such that $F \mid\left(a_{i-1}, a_{i}\right)$ is continuously differentiable with derivative $f_{i}>0$. Let us select any strictly decreasing sequence $\left(t_{k}\right)_{k \in \mathbb{N}}$ in $\left(0, F\left(a_{1-}\right)\right)$ which converges to $\inf \{F(x) \mid F(x)>$ $0\}$.

For any $k$, we can find a vector $\left(\alpha_{k o}, \ldots, \alpha_{k r}, \beta_{k 0}, \ldots, \beta_{k r}\right)$ satisfying

$$
\begin{gathered}
t_{k}=\alpha_{k 0}<\beta_{k 0}<F\left(a_{1-}\right) \quad \text { with } \quad F\left(a_{1-}\right)-\beta_{k 0}<\frac{1}{k}, \\
F\left(a_{i}\right)<\alpha_{k i}<\beta_{k i}<F\left(a_{(i+1)-}\right) \quad \text { with } \max \left\{\alpha_{k i}-F\left(a_{i}\right), F\left(a_{(i+1)-}\right)-\beta_{k i}\right\}<\frac{1}{k}
\end{gathered}
$$

for $i \in\{1, \ldots, r\}$. Setting $I_{k}:=\bigcup_{i=0}^{r}\left(\alpha_{k i}, \beta_{k i}\right)$, we consider the mapping

$$
\mathscr{D}_{n k}: \Psi \rightarrow \mathbb{R}^{\Omega}, \quad \psi \mapsto \sqrt{n} \int_{\mathbb{R}}\left[\Pi_{k}(\psi)\left(F_{n}(x)\right)-\Pi_{k}(\psi)(F(x))\right] \mathrm{d} x,
$$

where $\Pi_{k}(\psi):[0,1] \rightarrow[0,1]$ is defined via $\Pi_{k}(\psi)(t):=\int_{0}^{t} \mathbf{1}_{I_{k}}(u) \psi^{\prime}(u) \mathrm{d} u$.

The mapping $\mathscr{D}_{n k}$ may be viewed as a Borel random element of $\operatorname{UCB}(\Psi)$, following an analogous argument to that used for the mapping $\mathscr{D}_{n}$. We are now ready to formulate the auxiliary results which will be used to prove Theorems 3.1 and 4.1 . 
Proposition 5.2. Let the assumptions of either Theorem 3.1 or Theorem 4.1 be fulfilled. Then $\sup _{\psi \in \Psi}\left|\mathscr{D}_{n}(\psi)-\mathscr{D}_{n k}\right|$ is a real-valued random variable on $(\Omega, \mathcal{F}, P)$ for arbitrary $n, k \in \mathbb{N}$, and

$$
\lim _{k \rightarrow \infty} \limsup _{n \rightarrow \infty} \mathrm{P}\left(\left\{\sup _{\psi \in \Psi}\left|\mathscr{D}_{n}(\psi)-\mathscr{D}_{n k}(\psi)\right|>\varepsilon\right\}\right)=0
$$

holds for arbitrary $\varepsilon>0$.

Proposition 5.3. Let the assumptions of either Theorem 3.1 or Theorem 4.1 be fulfilled, and let $l^{\infty}(\mathbb{R})$ denote the set of bounded real-valued mappings on $\mathbb{R}$ which is equipped with the uniform metric. Then there exists some tight centered Gaussian Borel random element $B_{F}$ of $l^{\infty}(\mathbb{R})$ satisfying

$$
\mathrm{E}\left[B_{F}(x) B_{F}(y)\right]=F(x \wedge y)-F(x) F(y)+2 \sum_{k=1}^{\infty}\left(\mathrm{P}\left(X_{1} \leq x, X_{k} \leq y\right)-F(x) F(y)\right)
$$

for $x, y \in \mathbb{R}$ such that, for any $k \in \mathbb{N}$, the sequence $\left(\mathscr{D}_{n k}\right)_{n}$ converges in law to the centered Gaussian Borel random element $G_{k}$ of $\mathrm{UCB}(\Psi)$ defined by

$$
\begin{aligned}
& \mathrm{E}\left[G\left(\psi_{1}\right) G\left(\psi_{2}\right)\right] \\
& =\int_{\mathbb{R}^{2}} I_{k}(F(x)) \psi_{1}^{\prime}(F(x)) I_{k}(F(y)) \psi_{2}^{\prime}(F(y)) \\
& \quad \times\left[F(x \wedge y)-F(x) F(y)+2 \sum_{k=1}^{\infty}\left(\mathrm{P}\left(X_{1} \leq x, X_{k} \leq y\right)-F(x) F(y)\right)\right] \mathrm{d} x \mathrm{~d} y
\end{aligned}
$$

for every $\psi_{1}, \psi_{2} \in \Psi$.

Propositions 5.2 and 5.3 and Theorem 5.1 will be shown sequentially in the following section.

\section{Proofs of Propositions 5.2 and 5.3 and Theorem 5.1}

The assumptions and notation of Section 5 continue to hold; however, we want to carry out the announced proofs by considering the assumptions of Theorems 3.1 and 4.1 simultaneously. For that purpose, we will respectively replace (AI) and (AI') with the following assumption.

$\left(\mathrm{AI}^{*}\right)$ The distribution function $F$ fulfills

$$
\int_{q_{F}(0)}^{q_{F}(1)} F(x)^{-1 / 2-2 \delta}(1-F(x))^{1 / 2-\delta} \psi_{\rho}\left(\lambda F(x)^{1+\delta}\right) \mathrm{d} x<\infty
$$

for some $\lambda \in\left(0, \frac{1}{2}\right)$ and $\delta \in\left[0, \frac{1}{2}\right)$. For $\delta=0$, assumption (AI*) reduces to (AI), whereas we have (AI') if $\delta>0$.

The assumptions of independent $\left(X_{i}\right)_{i \in \mathbb{N}}$ or strictly stationary $\left(X_{i}\right)_{i \in \mathbb{N}}$ with mixing coefficients $(\alpha(i))_{i \in \mathbb{N}}$ satisfying assumption (AM) are described simultaneously in the following assumption.

$\left(\mathrm{AM}^{*}\right)$ The sequence $\left(X_{i}\right)_{i \in \mathbb{N}}$ is strictly stationary and strongly mixing with mixing coefficients $\alpha(i)$ satisfying

$$
\alpha(i) \leq \bar{\alpha}_{0} \exp \left(-\bar{\alpha}_{1} i\right), \quad i \in \mathbb{N},
$$

for some constants $\bar{\alpha}_{0} \geq 0$ and $\bar{\alpha}_{1}>0$.

In the case of independent $\left(X_{i}\right)_{i \in \mathbb{N}}$ we choose $\bar{\alpha}_{0}=0$. 
As a starting point, we conclude from $\left(\mathrm{AM}^{*}\right)$ that there is a centered Gaussian process $B_{F}:=\left(B_{F}(x)\right)_{x \in \mathbb{R}}$ satisfying

$$
\begin{aligned}
\operatorname{cov}\left(B_{F}(x), B_{F}(y)\right)= & \mathrm{E}\left[B_{F}(x) B_{F}(y)\right] \\
= & F(x \wedge y)-F(x) F(y) \\
& +2 \sum_{k=1}^{\infty}\left[\mathrm{P}\left(X_{1} \leq x, X_{k+1} \leq y\right)-F(x) F(y)\right],
\end{aligned}
$$

and which is a tight Borel random element of the space $D(\mathbb{R})$ of all càdlàg functions on $\mathbb{R}$ with respect to the supremum norm such that the sequence $\left(\left(\sqrt{n}\left[F_{n}(x)-F(x)\right]\right)_{x \in \mathbb{R}}\right)_{n}$, viewed as a sequence of Borel random elements of $D(\mathbb{R})$, converges in law to $B_{F}$ (see, e.g. [5, Corollary 1]). Moreover, the induced stochastic process $\left(B_{F}(x)\right)_{x \in \mathbb{R}}$ has paths which are continuous at every continuity point of $F$ (see Corollary 1 of [5] again).

Let $q_{F}(0)=: a_{0}<a_{1}<\cdots<a_{r+1}=: q_{F}(1)$ be as in the discussion preceding Proposition 5.2. Possibly changing to a suitable probability space, we may assume without loss of generality that there is a set $\left\{Z_{i j} \mid i \in \mathbb{N}, j \in\{0, \ldots, r+1\}\right\}$ of independent random variables all having the uniform distribution on $(0,1)$ as common distribution such that $\left\{Z_{i j} \mid i \in \mathbb{N}, j \in\{0, \ldots, r+1\}\right\}$ and $\left(X_{i}\right)_{i \in \mathbb{N}}$ are independent. This allows us to prove the following result on bounds for empirical distribution functions which will be crucial for our line of reasoning.

Lemma 6.1. Let assumptions ( $\left.A I^{*}\right)$ and $\left(A M^{*}\right)$ be satisfied, and let $\lambda \in\left(0, \frac{1}{2}\right)$ as well as $\delta \in$ $\left[0, \frac{1}{2}\right)$ be as in $\left(A I^{*}\right)$. The sample minimum of $\left(X_{1}, \ldots, X_{n}\right)$ will be denoted by $X_{n: 1}$. Then, for any $\eta \in(0,1)$ we find a constant $\gamma_{\eta} \in(0, \lambda)$, and a sequence $\left(A_{n \eta}\right)_{n \in \mathbb{N}}$ in $\mathcal{F}$ with $\mathrm{P}\left(A_{n \eta}\right) \geq 1-\eta$ such that

$$
\gamma_{\eta} \mathbf{1}_{\left[X_{n: 1}, 1\right]}(x) F(x)^{1+\delta} \mathbf{1}_{A_{n \eta}} \leq \mathbf{1}_{\left[X_{n: 1}, 1\right]}(x) F_{n}(x) \mathbf{1}_{A_{n \eta}}
$$

for any $x \in \mathbb{R}$.

Proof. Let $\left\{Z_{i j} \mid i \in \mathbb{N}, j \in\{0, \ldots, r+1\}\right\}$ be as discussed above. Then we may invoke the randomized probability integral transformation $U_{i}$ of each $X_{i}$, i.e.

$$
U_{i}:=F\left(X_{i}\right)-\sum_{j=0}^{r+1} \mathbf{1}_{\left\{a_{j}\right\}}\left(X_{i}\right) \mathrm{P}\left(\left\{X_{i}=a_{j}\right\}\right) Z_{i j} .
$$

In this way we obtain a strictly stationary sequence $\left(U_{i}\right)_{i \in \mathbb{N}}$ of random variables with the uniform distribution on $(0,1)$ as the common distribution and mixing coefficients $\alpha^{U}(i) \leq \bar{\alpha}_{0} \exp \left(-\bar{\alpha}_{1} i\right)$ with $\bar{\alpha}_{0}$ and $\bar{\alpha}_{1}$ as in $\left(\mathrm{AM}^{*}\right)$. Moreover, $X_{i}=q_{F}\left(U_{i}\right)$ a.s. so $F_{n}(x)=1 / n \sum_{i=1}^{n} \mathbf{1}_{(-\infty, F(x)]}\left(U_{i}\right)$ a.s. The statement of Lemma 6.1 is then a direct consequence of Inequality 12.11.2 of [31] if $\bar{\alpha}_{0}=0$, and it may be concluded from Theorem 1.3 of [26] otherwise.

Let us now turn to the proof of Proposition 5.2.

Proof of Proposition 5.2. Let $\lambda \in\left(0, \frac{1}{2}\right)$ and $\delta \in\left[0, \frac{1}{2}\right)$ be as in assumption (AI*). First, $\sup _{\psi \in \Psi}\left|\mathscr{D}_{n}(\psi)-\mathscr{D}_{n k}(\psi)\right|$ is a real-valued random variable as a continuous transformation of a Borel random element of $\mathrm{UCB}(\Psi)$. 
Since $\Psi$ is compact with respect to the uniform metric, it has some, at most countable, dense subset $\Psi_{0}$. Then we have $\sup _{\psi \in \Psi}\left|\mathscr{D}_{n}(\psi)-\mathscr{D}_{n k}(\psi)\right|=\sup _{\psi \in \Psi_{0}}\left|\mathscr{D}_{n}(\psi)-\mathscr{D}_{n k}(\psi)\right|$ because the paths of $\mathscr{D}_{n}$ and $\mathscr{D}_{n k}$ are continuous. In particular, for any fixed $\varepsilon \in(0,1)$,

$$
B_{n k \varepsilon}:=\left\{\sup _{\psi \in \Psi}\left|\mathscr{D}_{n}-\mathscr{D}_{n k}\right|>\varepsilon\right\}=\left\{\sup _{\psi \in \Psi_{0}}\left|\mathscr{D}_{n}-\mathscr{D}_{n k}\right|>\varepsilon\right\} \in \mathcal{F},
$$

and

$$
\begin{aligned}
g_{n k}(x) & :=\sqrt{n} \sup _{\psi \in \Psi_{0}}\left|\left[\psi\left(F_{n}(x)\right)-\psi(F(x))\right]-\left[\Pi_{k}(\psi)\left(F_{n}(x)\right)-\Pi_{k}(\psi)(F(x))\right]\right| \\
& =\sqrt{n} \sup _{\psi \in \Psi_{0}}\left|\int_{F(x)}^{F_{n}(x)} \mathbf{1}_{(0,1) \backslash I_{k}}(t) \psi^{\prime}(t) \mathrm{d} t\right|
\end{aligned}
$$

is indeed a random variable. The important part of the proof is to show the following statement.

(S) For any $\eta \in(0,1)$, there exist $C>0$ and a sequence $\left(A_{n \eta}\right)_{n \in \mathbb{N}}$ in $\mathcal{F}$ with $\mathrm{P}\left(A_{n \eta}\right) \geq 1-\eta$ such that

$$
\sqrt{\mathrm{E}\left[\left|\mathbf{1}_{A_{n \eta}} g_{n k}(x)\right|^{2}\right]} \leq C F(x)^{-1 / 2-2 \delta}(1-F(x))^{1 / 2-\delta} \psi_{\rho}\left(\lambda F(x)^{1+\delta}\right)
$$

for any $x \in\left(q_{F}(0), q_{F}(1)\right)$ and every $n \in \mathbb{N}$.

Let us first see how we can conclude the statement of Proposition 5.2 from $(\mathrm{S})$.

For arbitrary $\eta \in(0,1)$, choose $C,\left(A_{n \eta}\right)_{n \in \mathbb{N}}$ as in $(\mathrm{S})$, and, for $x \in\left(q_{F}(0), q_{F}(1)\right)$, use the notation $h(x):=F(x)^{-1 / 2-2 \delta}(1-F(x))^{1 / 2-\delta} \psi_{\rho}\left(\lambda F(x)^{1+\delta}\right)$. Then

$$
\int_{q_{F}(0)}^{q_{F}(1)} \mathrm{E}\left[\mathbf{1}_{A_{n \eta}} \mathbf{1}_{(0,1) \backslash I_{k}}(F(x)) g_{n k}(x)\right] \mathrm{d} x \leq C \int_{q_{F}(0)}^{q_{F}(1)} \mathbf{1}_{(0,1) \backslash I_{k}}(F(x)) h(x) \mathrm{d} x
$$

and

$$
\left(\mathrm{E}\left[\left|\mathbf{1}_{A_{n \eta}} g_{n k}(x)\right|\right]\right)^{2} \leq \mathrm{E}\left[\left|\mathbf{1}_{A_{n \eta}} g_{n k}(x)\right|^{2}\right] \leq C^{2} h(x)^{2} \quad \text { for any } x \in\left(q_{F}(0), q_{F}(1)\right) .
$$

By the continuous mapping theorem, the convergence in law of $\left(\sqrt{n}\left[F_{n}-F\right]\right)_{n \in \mathbb{N}}$ implies the convergence in law of $\left(\sup _{x \in \mathbb{R}}\left|\sqrt{n}\left[F_{n}(x)-F(x)\right]\right|\right)_{n \in \mathbb{N}}$. In particular, the latter sequence is uniformly tight, which implies that, for every $\beta \in(0,1)$, there exists some $A_{\beta} \in \mathcal{F}$ with $\mathrm{P}\left(A_{\beta}\right) \geq 1-\beta$ such that $\left(\mathbf{1}_{A_{\beta}} \sup _{x \in \mathbb{R}}\left|\left[F_{n}(x)-F(x)\right]\right|\right)_{n \in \mathbb{N}}$ converges uniformly to 0 . Since any $I_{k}$ is a finite union of open intervals of $\mathbb{R}$, it is then easy to verify that $\left(\mathbf{1}_{I_{k}}(F(x)) g_{n k}(x)\right)_{n \in \mathbb{N}}$ converges in probability to 0 for any $x \in\left(q_{F}(0), q_{F}(1)\right)$. Moreover, (6.3) means that $\left(\mathbf{1}_{I_{k}}(F(x)) g_{n k}(x) \mathbf{1}_{A_{n \eta}}\right)_{n \in \mathbb{N}}$ is uniformly integrable for $x \in\left(q_{F}(0), q_{F}(1)\right)$, implying that $\left(\mathbf{1}_{I_{k}}(F(x)) g_{n k}(x) \mathbf{1}_{A_{n \eta}}\right)_{n \in \mathbb{N}}$ converges in mean to 0 for $x \in\left(q_{F}(0), q_{F}(1)\right)$. Furthermore, by (6.3) and assumption (AI*), we can apply the dominated convergence theorem to obtain

$$
\lim _{n \rightarrow \infty} \int_{q_{F}(0)}^{q_{F}(1)} \mathrm{E}\left[\left|\mathbf{1}_{I_{k}}(F(x)) g_{n k}(x) \mathbf{1}_{A_{n \eta}}\right|\right] \mathrm{d} x=0 \quad \text { for } k \in \mathbb{N}, \eta \in(0,1) .
$$

Thus, by (6.2) and Markov's inequality, along with Tonelli's theorem,

$$
\lim _{k \rightarrow \infty} \limsup _{n \rightarrow \infty} \mathrm{P}\left(B_{n k \varepsilon} \cap A_{n \eta}\right) \leq \lim _{k \rightarrow \infty} \frac{2}{\varepsilon} \int_{q_{F}(0)}^{q_{F}(1)} \mathbf{1}_{(0,1) \backslash I_{k}}(F(x)) h(x) \mathrm{d} x .
$$


Furthermore, $\lim _{k \rightarrow \infty} \mathbf{1}_{(0,1) \backslash I_{k}}(F(x))=0$ for every $x \in\left(q_{F}(0), q_{F}(1)\right)$. Then in view of assumption (AI*) we can apply the dominated convergence theorem to conclude from (6.4) that

$$
\lim _{k \rightarrow \infty} \limsup _{n \rightarrow \infty} \mathrm{P}\left(B_{n k \varepsilon}\right) \leq \lim _{k \rightarrow \infty} \limsup _{n \rightarrow \infty} \mathrm{P}\left(B_{n k \varepsilon} \cap A_{n \eta}\right)+\eta=\eta .
$$

So it remains to show $(\mathrm{S})$.

Proof of $(S)$. For $\eta \in(0,1)$, choose $C_{\eta}>0, \gamma_{\eta} \in(0, \lambda)$, and $\left(A_{n \eta}\right)_{n \in \mathbb{N}}$ as in Lemma 6.1. First, since every $\psi \in \Psi$ is concave with $\psi(0)=0$, we have $\psi\left(\lambda F(x)^{1+\delta}\right) \geq \lambda F(x)^{\delta} \psi(F(x))$. Hence,

$$
\sup _{\psi \in \Psi_{0}} \psi(F(x)) \leq \frac{1}{\lambda} F(x)^{-\delta} \sup _{\psi \in \Psi_{0}} \psi\left(\lambda F(x)^{1+\delta}\right) \leq \frac{2}{\gamma_{\eta}} F(x) \frac{\psi_{\rho}\left(\lambda F(x)^{1+\delta}\right)}{F(x)^{1+\delta}}
$$

for $x \in\left(q_{F}(0), q_{F}(1)\right)$. Then we obtain, for $\omega \in A_{n \eta}$ and $q_{F}(0)<x<X_{n: 1}(\omega)$ with $X_{n: 1}(\omega):=\min _{i \in\{1, \ldots, n\}} X_{i}(\omega)$,

$$
g_{n k}(x)(\omega) \leq \sqrt{n} \sup _{\psi \in \Psi_{0}} \Psi(F(x)) \leq \frac{2}{\gamma_{\eta}} \sqrt{n}\left|F_{n}(x)(\omega)-F(x)\right| \frac{\psi_{\rho}\left(\lambda F(x)^{1+\delta}\right)}{F(x)^{1+\delta}} .
$$

Since the right-sided derivative of any $\psi \in \Psi$ is nonincreasing, we can conclude, from Lemma 6.1 along with (5.2), that

$$
\begin{aligned}
g_{n k}(x)(\omega) & \leq \sqrt{n} \sup _{\psi \in \Psi_{0}}\left|\int_{F(x)}^{F_{n}(x)(\omega)} \psi^{\prime}(t) \mathrm{d} t\right| \\
& \leq \sqrt{n}\left|F_{n}(x)(\omega)-F(x)\right| \sup _{\psi \in \Psi_{0}} \psi^{\prime}\left(\gamma_{\eta} F(x)^{1+\delta}\right) \\
& \leq \sqrt{n}\left|F_{n}(x)(\omega)-F(x)\right| \frac{2 \psi_{\rho}\left(\left(\gamma_{\eta} / 2\right) F(x)^{1+\delta}\right)}{\gamma_{n} F(x)^{1+\delta}} \\
& \leq \frac{2}{\gamma_{\eta}} \sqrt{n}\left|F_{n}(x)(\omega)-F(x)\right| \frac{\psi_{\rho}\left(\lambda F(x)^{1+\delta}\right)}{F(x)^{1+\delta}}
\end{aligned}
$$

for $\omega \in A_{n \eta}$ and $F(x) \geq X_{n: 1}(\omega)$.

Finally, by Lemma C.1 (see Appendix C), we can find a constant $\bar{C}>0$ such that

$$
\mathrm{E}\left[n\left[F_{n}(x)-F(x)\right]^{2}\right] \leq \bar{C}^{2}[F(x)(1-F(x))]^{1-2 \delta}
$$

holds for any $x \in\left(q_{F}(0), q_{F}(1)\right)$. Setting $C:=(2 \bar{C}) / \gamma_{\eta}$, then (S) follows immediately from (6.5) and (6.6). The proof of Proposition 5.2 is complete.

Proof of Proposition 5.3. Lemma A.1 (see Appendix A) gives the following representation of $\mathscr{D}_{n k}$ :

$$
\mathscr{D}_{n k}(\psi)=-\sum_{i=0}^{r} \int_{\alpha_{k i}}^{\beta_{k i}} \sqrt{n}\left[q_{F_{n}}(t)-q_{F}(t)\right] \psi^{\prime}(t) \mathrm{d} t \quad \text { for } k \in \mathbb{N}, \psi \in \Psi .
$$

Here $q_{F_{n}}$ denotes the left-continuous quantile function of $F_{n}$. Representation (6.7) suggests applying already known asymptotic results for the quantile processes $\left(\sqrt{n}\left[q_{F_{n}}(t)-q_{F}(t)\right]\right)_{t \in(0,1)}$.

First, we already have convergence in law of $\left(\sqrt{n}\left[F_{n}-F\right]\right)_{n \in \mathbb{N}}$ to some tight centered Borel random element $B_{F}$ of $D(\mathbb{R})$ with covariance function satisfying (6.1), and whose paths are 
continuous at every continuity point of $F$. Furthermore, by construction, we can find, for any $k \in \mathbb{N}$, some positive constant $\varepsilon_{k}>0$ such that $F \mid\left(q_{F}\left(\alpha_{k i}\right)-\varepsilon_{k}, q_{F}\left(\beta_{k i}\right)+\varepsilon_{k}\right)$ is continuously differentiable with derivative $f_{k i}>0$ for $i=0, \ldots, r$.

Before proceeding, we need some notation. Setting $a_{k i}:=q_{F}\left(\alpha_{k i}\right)-\varepsilon_{k}$ and $b_{k i}:=q_{F}\left(\beta_{k i}\right)+$ $\varepsilon_{k}$, we denote the real vector space of restrictions of members of $D(\mathbb{R})$ to $J_{k}:=\bigcup_{i=0}^{r}\left[a_{k i}, b_{k i}\right]$ by $D\left(J_{k}\right)$, and we endow it with the supremum norm. The subset $D_{1}\left(J_{k}\right) \subseteq D\left(J_{k}\right)$ is defined to consist of all restrictions of distribution functions on $\mathbb{R}$ to $J_{k}$. Finally, $l^{\infty}\left(\left[\alpha_{k 0}, \beta_{k 0}\right]\right) \times \cdots \times$ $l^{\infty}\left(\left[\alpha_{k r}, \beta_{k r}\right]\right)$ stands for the set of mappings $\left(g_{0}, \ldots, g_{r}\right):\left[\alpha_{k 0}, \beta_{k 0}\right] \times \cdots \times\left[\alpha_{k r}, \beta_{k r}\right] \rightarrow$ $\mathbb{R}^{r+1}$ whose components are bounded. It will be equipped with the metric $d$, defined by $d\left(\left(g_{0}, \ldots, g_{r}\right),\left(h_{0}, \ldots, h_{r}\right)\right):=\sum_{i=0}^{r} \sup _{t \in\left[\alpha_{k i}, \beta_{k i}\right]}\left|g_{i}(t)-h_{i}(t)\right|$.

Next, it follows from the continuous mapping theorem that $\left(\sqrt{n}\left[F_{n}-F\right] \mid J_{k}\right)_{n \in \mathbb{N}}$, as a sequence of Borel random elements of $D\left(J_{k}\right)$, converges in law to the tight centered Gaussian Borel random element $B_{F k}:=B_{F} \mid J_{k}$ which has continuous paths. Therefore, in view of Lemma 21.4 of [32], we can apply the functional delta method (see Theorem 20.8 of [32]) to the mapping $\Phi_{k}: D_{1}\left(J_{k}\right) \rightarrow l^{\infty}\left(\left[\alpha_{k 0}, \beta_{k 0}\right]\right) \times \cdots \times l^{\infty}\left(\left[\alpha_{k r}, \beta_{k r}\right]\right)$, defined by

$$
\Phi_{k}\left(G \mid J_{k}\right):=\left(q_{G}\left|\left[\alpha_{k 0}, \beta_{k 0}\right], \ldots, q_{G}\right|\left[\alpha_{k r}, \beta_{k r}\right]\right)
$$

with $q_{G}$ denoting the left-continuous quantile function of $G$, to conclude that the sequence $\left(\sqrt{n}\left[\Phi_{k}\left(F_{n} \mid J_{k}\right)-\Phi_{k}\left(F \mid J_{k}\right)\right]\right)_{n \in \mathbb{N}}$ converges in law to the tight Borel random element

$$
\left(-\frac{B_{F}}{f_{k 0}} \circ q_{F}\left|\left[\alpha_{k 0}, \beta_{k 0}\right], \ldots,-\frac{B_{F}}{f_{k r}} \circ q_{F}\right|\left[\alpha_{k r}, \beta_{k r}\right]\right) .
$$

Then, by (6.7), it follows from application of the continuous mapping theorem that $\left(\mathscr{D}_{n k}\right)_{n \in \mathbb{N}}$ converges in law to some tight Borel random element $G_{k}$ of $l^{\infty}(\Psi)$, defined by

$$
G_{k}(\psi):=\sum_{i=0}^{r} \int_{\alpha_{k i}}^{\beta_{k i}} \frac{B_{F}\left(q_{F}(t)\right)}{f_{k i}\left(q_{F}(t)\right)} \psi^{\prime}(t) \mathrm{d} t .
$$

Since, by construction, $F \mid\left(a_{k i}, b_{k i}\right)$ is invertible for every $i \in\{0, \ldots, r\}$, we obtain, by a change of variable formula,

$$
\begin{aligned}
G_{k}(\psi) & =\int_{\mathbb{R}} B_{F}(x) \sum_{i=0}^{r} \mathbf{1}_{\left(q_{F}\left(\alpha_{k i}\right), q_{F}\left(\beta_{k i}\right)\right)}(x) \psi^{\prime}(F(x)) \mathrm{d} x \\
& =\int_{\mathbb{R}} B_{F}(x) \mathbf{1}_{I_{k}}(F(x)) \psi^{\prime}(F(x)) \mathrm{d} x .
\end{aligned}
$$

Moreover, the set of Borel probability measures on $\operatorname{UCB}(\Psi)$ is a Polish space because $\operatorname{UCB}(\Psi)$, equipped with the uniform metric, is a Polish space too. Since, each $\mathscr{D}_{n k}$ is a Borel random element of $\operatorname{UCB}(\Psi)$, the stochastic process $\left(G_{k}(\psi)\right)_{\psi \in \Psi}$ has continuous paths a.s., and then $G_{k}$ is as required.

Theorem 5.1 may be concluded from Propositions 5.2 and 5.3 in the following way.

Proof of Theorem 5.1. Let $l^{\infty}(\mathbb{R})$ be the space of bounded real-valued mappings on $\mathbb{R}$ which is equipped with the uniform metric. Furthermore, let $B_{F}$ be the Gaussian Borel random element of $l^{\infty}(\mathbb{R})$ from Proposition 5.3, inducing a sequence $\left(G_{k}\right)_{k \in \mathbb{N}}$ of Gaussian Borel random elements of $\mathrm{UCB}(\Psi)$ as in Proposition 5.3. 
Since the mappings $\mathscr{D}_{n}$ and $\mathscr{D}_{n k}$ are Borel random elements of a separable metric space, we can apply Theorem 4.2 of [7]. Therefore, in view of Propositions 5.2 and 5.3 it remains to show that the mapping $G(\psi):=\int_{q_{F}(0)}^{q_{F}(1)} \psi^{\prime}(F(x)) B_{F}(x) \mathrm{d} x$ defines a Borel random element $G$ of $\operatorname{UCB}(\Psi)$ such that $\left(G_{k}\right)_{k}$ converges in law to $G$.

Let $\lambda, \delta \in\left[0, \frac{1}{2}\right.$ ), as in condition (AI*). Then, by Lemma C.1 (see Appendix C), there exists some constant $C \geq 0$ such that $\operatorname{var}\left(B_{F}(x)\right) \leq C^{2}\left[F(x)(1-F(x)]^{1-2 \delta}\right.$ for every $x \in \mathbb{R}$. Then we can conclude, from (5.2) along with $\left(\mathrm{AI}^{*}\right)$, that

$$
\begin{aligned}
& \int_{q_{F}(0)}^{q_{F}(1)} \sqrt{\operatorname{var}\left(\psi^{\prime}(F(x)) B_{F}(x)\right)} \mathrm{d} x \\
& \leq \int_{q_{F}(0)}^{q_{F}(1)} \sqrt{\operatorname{var}\left(\psi^{\prime}\left(2 \lambda F(x)^{1+\delta}\right) B_{F}(x)\right)} \mathrm{d} x \\
& \quad \stackrel{(5.2)}{\leq} \frac{2}{\lambda} \int_{q_{F}(0)}^{q_{F}(1)} \sqrt{\operatorname{var}\left(\frac{\psi_{\rho}\left(\lambda F(x)^{1+\delta}\right)}{F(x)^{1+\delta}} B_{F}(x)\right)} \mathrm{d} x \\
& \quad \leq \frac{2 C}{\lambda} \int_{q_{F}(0)}^{q_{F}(1)} F(x)^{-1 / 2-2 \delta}(1-F(x))^{1 / 2-\delta} \psi_{\rho}\left(\lambda F(x)^{1+\delta}\right) \mathrm{d} x \\
& \quad \stackrel{\left(\mathrm{AI}^{*}\right)}{<} \infty .
\end{aligned}
$$

By Lemma 3.3 of [27], this means that $B_{F}$ has paths in $V$ a.s., where $V$ denotes the set of all $g \in l^{\infty}(\mathbb{R})$ such that $g \psi_{\rho}\left(\lambda F^{1+\delta}\right) / F^{1+\delta}$ is integrable with respect to the ordinary LebesgueBorel measure $\lambda^{1}$ on $\mathbb{R}$. By the same argument from [27], $(G(\psi))_{\psi \in \Psi}$ is a well-defined centered Gaussian process. Moreover, $\lim _{k \rightarrow \infty} \mathbf{1}_{(0,1) \backslash I_{k}}(F(x))=0$ holds for every $x \in\left(q_{F}(0), q_{F}(1)\right)$. Then, an application of the dominated convergence theorem along with (5.2) yields

$$
\begin{aligned}
\lim _{k \rightarrow \infty} \sup _{\psi \in \Psi}\left|G(\psi)-G_{k}(\psi)\right| & \leq \lim _{k \rightarrow \infty} \int_{q_{F}(0)}^{q_{F}(1)} \mathbf{1}_{(0,1) \backslash I_{k}}(F(x))\left|B_{F}(x) \frac{\psi_{\rho}\left(\lambda F(x)^{1+\delta}\right)}{\lambda F(x)^{1+\delta}}\right| \mathrm{d} x \\
& =0 \quad \text { a.s. }
\end{aligned}
$$

Since every process $\left(G_{k}(\psi)\right)_{\psi \in \Psi}$ has paths in $\operatorname{UCB}(\Psi),(6.8)$ tells us that $(G(\psi)) \psi \in \Psi$ has paths in $\operatorname{UCB}(\Psi)$ a.s. So we may choose an indistinguishable version of $(G(\psi))_{\psi \in \Psi}$ as a centered Gaussian Borel random element of $\operatorname{UCB}(\Psi)$, denoted by $G$, which is in addition tight because the uniform topology on $\mathrm{UCB}(\Psi)$ is separably and completely metrizable. Finally, (6.8) also implies that $\left(G_{k}\right)_{k \in \mathbb{N}}$ converges in law to $G$. The proof is now complete.

\section{Proof of the main results}

We maintain the notions and notation from Sections 3 and 4.

First, assumption (AC) on the risk measure $\rho$ allows us to apply Proposition 5.1. Therefore, $\rho=\sup _{\psi \in \Psi} \rho_{\psi}$ for some set $\Psi$ of continuous, concave distortion functions which is compact with respect to the uniform metric on $[0,1]^{[0,1]}$. The compactness of $\Psi$ implies, by an application of the dominated convergence theorem along with Lemma 5.1, that

$S(\rho(F)):=\left\{\psi \in \Psi \mid \rho(F)=\rho_{\psi}(F)\right\} \neq \varnothing$ and compact with respect to the uniform metric

under (AI) or (AI'). 
Now, let $\left(\mathscr{D}_{n}\right)_{n \in \mathbb{N}}$ be the sequence of Borel random elements of $\operatorname{UCB}(\Psi)$ defined as in Section 5. Each can be decomposed in the following way:

$$
D_{n}(\psi)=\sqrt{n}\left[\rho_{\psi}\left(F_{n}\right)-\rho_{\psi}(F)\right] .
$$

According to Theorem 5.1, if the assumptions of either Theorem 3.1 or Theorem 4.1 are satisfied, then there exists a tight centered Gaussian Borel random element $G$ of $\operatorname{UCB}(\Psi)$ with

$$
\begin{aligned}
& \mathrm{E}\left[G\left(\psi_{1}\right) G\left(\psi_{2}\right)\right] \\
& =\int_{\mathbb{R}^{2}} \psi_{1}^{\prime}(F(x)) \psi_{2}^{\prime}(F(y)) \\
& \quad \times\left[F(x \wedge y)-F(x) F(y)+2 \sum_{k=1}^{\infty}\left(\mathrm{P}\left(X_{1} \leq x, X_{k} \leq y\right)-F(x) F(y)\right)\right] \mathrm{d} x \mathrm{~d} y
\end{aligned}
$$

for any $\psi_{1}, \psi_{2} \in \Psi$ such that $\left(\mathscr{D}_{n}(\psi)\right)_{\psi \in \Psi}$ converges in law to $G$. As a further consequence, (7.2) along with the representation $\rho=\sup _{\psi \in \Psi} \rho_{\psi}$ and (7.1) allows us to apply the functional delta method for supremum functionals (cf. [28]) to conclude that $\left(\sqrt{n}\left[\rho_{n}(F)-\rho(F)\right]\right)_{n \in \mathbb{N}}$ converges in law to $\sup _{\psi \in S(\rho)} G_{\psi}$. Finally, if $\mathrm{E}\left[\left(G\left(\psi_{1}\right)-G\left(\psi_{2}\right)\right)^{2}\right] \neq 0$ for different $\psi_{1}, \psi_{2} \in$ $S(\rho(F))$, then it is well known that the paths of $G \mid S(\rho(F))$ have unique maximizers a.s. (cf. [17]). Then by measurable selection we can find a Borel random element $Z$ of $S(\rho(F))$ such that $G(Z)$ is distributed as $\sup _{\psi \in S(\rho)} G_{\psi}$. This completes the proof.

\section{Appendix A}

Lemma A.1. Let $\psi:[0,1] \rightarrow[0,1]$ be a nondecreasing, continuous mapping with $\psi(0)=$ 0 , and let $G$ be any distribution function on $\mathbb{R}$ such that $\int_{-\infty}^{0} \psi(G(x)) \mathrm{d} x$ and $\int_{0}^{\infty}[\psi(1)-$ $\psi(G(x))] \mathrm{d} x$ are finite. Then

$$
\int_{-\infty}^{0} \psi(G(x)) \mathrm{d} x-\int_{0}^{\infty}[\psi(1)-\psi(G(x))] \mathrm{d} x=-\int_{0}^{1} q_{G} \mathrm{~d} \mu_{\psi},
$$

where $q_{G}$ and $\mu_{\psi}$ respectively denote the left-continuous quantile function of $G$ and the Borel probability measure on $[0,1]$ induced by $\psi$.

Proof. Let $\mu_{\psi \circ G}$ denote the Borel probability measure on $\mathbb{R}$ induced by the right-continuous mapping $\psi \circ G$. It coincides with the image measure of $\mu_{\psi}$ under $q_{G}$, implying that

$$
\int_{0}^{1} q_{G} \mathrm{~d} \mu_{\psi}=\int_{\mathbb{R}} x \mu_{\psi \circ G}(\mathrm{~d} x) .
$$

Furthermore, by the right continuity of $\psi \circ G$,

$$
\begin{aligned}
\int_{-\infty}^{0} x \mu_{\psi \circ G}(\mathrm{~d} x) & =-\int_{\mathbb{R}}\left[-\mathbf{1}_{(-\infty, 0]}(x) x\right] \mu_{\psi \circ G}(\mathrm{~d} x) \\
& =-\int_{0}^{\infty} \mu_{\psi \circ G}((-\infty,-\beta]) \mathrm{d} \beta \\
& =-\int_{0}^{\infty} \psi(G(-\beta)) \mathrm{d} \beta
\end{aligned}
$$


and

$$
\begin{aligned}
\int_{0}^{\infty} x \mu_{\psi \circ G}(\mathrm{~d} x) & =\int_{\mathbb{R}} \mathbf{1}_{(0, \infty)}(x) x \mu_{\psi \circ G}(\mathrm{~d} x) \\
& =\int_{0}^{\infty} \mu_{\psi \circ G}((\beta, \infty)) \mathrm{d} \beta \\
& =\int_{0}^{\infty}[\psi(1)-\psi(G(\beta))] \mathrm{d} \beta .
\end{aligned}
$$

The statement of Lemma A.1 follows from (A.1) and (A.2) upon applying a change of variable formula to (A.1).

\section{Appendix B. Proof of Proposition 5.1}

The proof of Proposition 5.1 relies on the following lemma.

Lemma B.1. If, in addition, $X \wedge Y, X \vee Y \in \mathcal{X}$ for $X, Y \in \mathcal{X}$ then the first property of assumption (AC) implies that

$$
\rho(X)=\sup _{m \in \mathbb{N}} \inf _{k \in \mathbb{N}} \rho\left(F_{\left[X^{+} \wedge k\right]-\left[X^{-} \wedge m\right]}\right) \quad \text { for all } X \in \mathcal{X} .
$$

Proof. In view of Proposition 6.6 of [15] the first property of assumption (AC) allows us to apply Lemma 6.5 from the same paper. According to part 1 of Lemma 6.5, we have $\sup _{m \in \mathbb{N}} \rho\left(F_{X^{+}-\left[X^{-} \wedge m\right]}\right)=\rho\left(F_{X}\right)$, whereas $\inf _{k \in \mathbb{N}} \rho\left(F_{\left[X^{+} \wedge k\right]-\left[X^{-} \wedge m\right]}\right)=\rho\left(F_{X^{+}-\left[X^{-} \wedge m\right]}\right)$ holds for any $m \in \mathbb{N}$ due to part 2 of Lemma 6.5. The statement of Lemma B.1 obviously follows.

Lemma B.1 enables us to conclude a robust representation of $\rho$ by concave distortion risk measures when its restriction to $\left\{F_{X} \mid X \in \mathcal{L}^{\infty}(\Omega, \mathcal{F}, \mathrm{P})\right\}$ admits such a representation.

Lemma B.2. Let $\Psi$ be any set of concave distortion functions such that

$$
\rho\left(F_{X}\right)=\sup _{\psi \in \Psi} \rho_{\psi}\left(F_{X}\right)
$$

holds for $X \in \mathcal{L}^{\infty}(\Omega, \mathcal{F}, \mathrm{P})$.

If $X \wedge Y, X \vee Y \in \mathcal{X}$ for $X, Y \in \mathcal{X}$, and if $\rho$ satisfies the first property of assumption (AC), then $\rho(F)=\sup _{\psi \in \Psi} \rho_{\psi}(F)$ is valid for arbitrary $F \in \mathbb{F} X$.

Proof. Set $\tilde{\rho}:=\sup _{\psi \in \Psi} \rho_{\psi}$. The proof is divided into two steps. In the first step we will show that $\widetilde{\rho}$ is well defined and defines a law-invariant coherent risk measure on $\mathbb{F} X$, which obviously coincides with $\rho$ on $\left\{F_{X} \mid X \in \mathscr{L}^{\infty}(\Omega, \mathcal{F}, \mathrm{P})\right\}$. In the second step we will prove that both risk measures are identical.

Step 1. If we can show that $\rho_{\psi}\left(F_{X}\right) \in \mathbb{R}$ (for all $\psi \in \Psi$ ) and $\sup _{\psi \in \Psi} \rho_{\psi}\left(F_{X}\right)<\infty$ for all $X \in \mathcal{X}$, then it follows easily that $\tilde{\rho}$ defines a law-invariant coherent risk measure on $\mathcal{X}$, since every concave distortion risk measure $\rho_{\psi}$ is a law-invariant coherent risk measure. Of course, the mentioned conditions hold if we can show that, for all $X \in \mathcal{X}$ with distribution function $F_{X}$,

$$
\sup _{\psi \in \Psi} \int_{-\infty}^{0} \psi\left(F_{X}(x)\right) \mathrm{d} x \leq \rho\left(F_{-X^{-}}\right)
$$

and, for all $\psi \in \Psi, \quad \int_{0}^{\infty}\left[1-\psi\left(F_{X}(x)\right)\right] \mathrm{d} x<\infty$. 
To verify the first statement of (B.1), we choose $X \in \mathcal{X}$. For every $\psi \in \Psi$, we have

$$
\psi\left(F_{-X^{-}}(x)\right) \leq \liminf _{m \rightarrow \infty} \psi\left(F_{-\left[X^{-} \wedge m\right]}(x)\right)
$$

at every continuity point $x<0$ of the distribution function $F_{-X^{-}}$of $-X^{-}$, since $\psi$ as a concave function is lower semicontinuous. Using this and applying Fatou's lemma, we obtain

$$
\begin{aligned}
\sup _{\psi \in \Psi} \int_{-\infty}^{0} \psi\left(F_{X}(x)\right) \mathrm{d} x & \leq \sup _{\psi \in \Psi} \int_{-\infty}^{0} \psi\left(F_{-X^{-}}(x)\right) \mathrm{d} x \\
& \leq \sup _{\psi \in \Psi} \int_{-\infty}^{0} \liminf _{m \rightarrow \infty} \psi\left(F_{-\left[X^{-} \wedge m\right]}(x)\right) \mathrm{d} x \\
& \leq \sup _{\psi \in \Psi} \liminf _{m \rightarrow \infty} \int_{-\infty}^{0} \psi\left(F_{-\left[X^{-} \wedge m\right]}(x)\right) \mathrm{d} x \\
& =\sup _{\psi \in \Psi} \liminf _{m \rightarrow \infty} \rho_{\psi}\left(F_{-\left[X^{-} \wedge m\right]}\right) \\
& \leq \liminf _{m \rightarrow \infty} \rho\left(F_{-\left[X^{-} \wedge m\right]}\right) \\
& \leq \rho\left(F_{-X^{-}}\right) .
\end{aligned}
$$

Hence, the first statement of (B.1) indeed holds. To verify the second statement of (B.1), we choose $X \in X$. As $\psi$ is nondecreasing and concave, its restriction to $(0,1]$ is continuous, so that

$$
1-\psi\left(F_{X}(x)\right)=\psi(1)-\psi\left(F_{X}(x)\right) \leq \psi^{\prime}\left(F_{X}\left(x_{0}\right)\right)\left[1-F_{X}(x)\right] \text { for all } x \geq x_{0},
$$

for any $x_{0}>0$ such that $F_{X}\left(x_{0}\right)>0$. Moreover, the integral $\int_{0}^{\infty}\left[1-F_{X}(x)\right] \mathrm{d} x$ exists since $X \in \mathcal{L}^{1}(\Omega, \mathcal{F}, \mathrm{P})$. Hence,

$$
\int_{0}^{\infty}\left[1-\psi\left(F_{X}(x)\right)\right] \mathrm{d} x \leq \int_{0}^{x_{0}}\left[1-\psi\left(F_{X}(t)\right)\right] \mathrm{d} x+\psi^{\prime}\left(F_{X}\left(x_{0}\right)\right) \int_{x_{0}}^{\infty}\left[1-F_{X}(x)\right] \mathrm{d} x<\infty .
$$

This shows that the second statement of (B.1) holds too.

Step 2. The first property of assumption (AC) on $\rho$ ensures that the right-hand side of

$$
0 \leq \widetilde{\rho}\left(F_{-(X-r)^{+}}\right) \stackrel{(\text { B.1 })}{\leq} \rho\left(F_{-(X-r)^{+}}\right)
$$

converges to 0 as $r \rightarrow \infty$ for every nonnegative $X \in X$. Therefore, the first property of assumption (AC) is fulfilled by $\widetilde{\rho}$ too, and Lemma B.1 applied to $\widetilde{\rho}$ implies that $\rho=\widetilde{\rho}$ on $\mathcal{X}$. The proof is now complete.

We are now ready to prove Proposition 5.1.

Proof of Proposition 5.1. Possibly changing to a suitable probability space we assume that $L^{2}(\Omega, \mathcal{F}, \mathrm{P})$ is separable. Then in the specified setting, Corollary 4.78 of [11] along with Theorem 2.1 of [13] yield the existence of some set $\widetilde{\Psi}$ of concave distortions such that $\rho\left(F_{X}\right)=$ $\sup _{\psi \in \widetilde{\Psi}} \rho_{\psi}\left(F_{X}\right)$ holds for $X \in \mathcal{L}^{\infty}(\Omega, \mathcal{F}, \mathrm{P})$. Note that all members of the topological closure $\Psi$ of $\widetilde{\Psi}$ with respect to the uniform metric are again concave distortion functions. Therefore, in view of (5.1),

$$
|\psi(q)-\psi(p)| \leq \psi_{\rho}(q-p) \text { for } \psi \in \Psi \text { and } 0 \leq p<q \leq 1 .
$$


Since $\lim _{p \rightarrow 0_{+}} \psi_{\rho}(p)=0$ by the second property of assumption (AC), we conclude that $\Psi$ is uniformly equicontinuous with respect to the uniform metric, which means that, by the ArzelaAscoli theorem, it is not only closed but also compact with respect to the supremum metric. We want to show that $\rho$ admits a robust representation by concave distortion risk measures with concave distortions from $\Psi$. For this purpose, by Lemma B.2, it suffices to show that $\rho\left(F_{X}\right)=\sup _{\psi \in \Psi} \rho_{\psi}\left(F_{X}\right)$ is valid for every $X \in \mathcal{L}^{\infty}(\Omega, \mathcal{F}, \mathrm{P})$.

Indeed, for any fixed $X \in \mathcal{L}^{\infty}(\Omega, \mathcal{F}, \mathrm{P})$ with distribution function $F_{X}$, there exists some $\varepsilon>0$ such

$$
\left.\rho_{\psi}\left(F_{X}\right)=\int_{-\varepsilon}^{0} \psi\left(F_{X}\right)(x)\right) \mathrm{d} x-\int_{0}^{\varepsilon}\left[1-\psi\left(F_{X}(x)\right)\right] \mathrm{d} x \quad \text { for all } \psi \in \Psi .
$$

Then a routine application of the dominated convergence theorem yields the continuity of the mapping

$$
\Phi: \Psi \rightarrow \mathbb{R}, \quad \psi \mapsto \rho_{\psi}\left(F_{X}\right)
$$

with respect to the uniform metric. Therefore, $\lim _{k \rightarrow \infty} \rho_{\psi_{k}}\left(F_{X}\right)=\rho_{\psi}\left(F_{X}\right)$ holds for any sequence $\left(\psi_{k}\right)_{k \in \mathbb{N}}$ in $\widetilde{\Psi}$ which converges to some $\psi$ with respect to the uniform metric. Hence, obviously, $\rho\left(F_{X}\right)=\sup _{\psi \in \Psi} \rho_{\psi}\left(F_{X}\right)$, and, thus, $\rho=\sup _{\psi \in \Psi} \rho_{\psi}$ due to Lemma B.2. The proof is complete.

\section{Appendix C}

Lemma C.1. Let $\left(Z_{i}\right)_{i \in \mathbb{N}}$ be a strictly stationary, strongly mixing sequence of random variables on some probability space $(\widetilde{\Omega}, \tilde{\mathcal{F}}, \widetilde{\mathrm{P}})$ with common distribution function $H$ and mixing coefficients $\alpha(i)$ satisfying

$$
\alpha(i) \leq \bar{\alpha}_{0} \exp \left(-\bar{\alpha}_{1} i\right), \quad i \in \mathbb{N},
$$

for some constants $\bar{\alpha}_{0}>0$ and $\bar{\alpha}_{1}>0$. Then, for any $\eta \in(0,1)$, there exists some constant $C_{\eta}$ such that

$$
H(x)(1-H(x))+2 \sum_{i=1}^{\infty}\left[\widetilde{\mathrm{P}}\left(\left\{Z_{1} \leq x, Z_{i+1} \leq x\right\}\right)-H(X)^{2}\right] \leq C_{\eta}[H(x)(1-H(x))]^{1-\eta}
$$

for every $x \in \mathbb{R}$.

Proof. Let $\eta \in(0,1)$ and $x \in \mathbb{R}$, and define $Y_{i}(x):=\mathbf{1}_{(-\infty, x]} \circ Z_{i}$. Without loss of generality, we assume that $\bar{\alpha}_{0} \geq 1$.

First, observe that, for any $n \geq 2$,

$$
\begin{aligned}
\frac{1}{n} \operatorname{var}\left(\sum_{i=1}^{n} Y_{i}(x)\right)= & \operatorname{var}\left(Z_{1}\right)+2 \sum_{i=1}^{n-1} \frac{n-i}{n} \operatorname{cov}\left(Z_{1}, Z_{i+1}\right) \\
= & H(x)(1-H(x)) \\
& +2 \sum_{i=1}^{n-1} \frac{n-i}{n}\left[\widetilde{\mathrm{P}}\left(\left\{Z_{1} \leq x, Z_{i+1} \leq x\right\}\right)-H(x)^{2}\right]
\end{aligned}
$$


By the assumption on $(\alpha(i))_{i \in \mathbb{N}}$, the series $\sum_{i=1}^{\infty} \operatorname{cov}\left(Z_{i}, Z_{i+1}\right)$ converges absolutely (cf., e.g. [2, Proposition 16.3.1]), so, by the dominated convergence theorem,

$$
\begin{aligned}
\lim _{n \rightarrow \infty} \frac{1}{n} \operatorname{var}\left(\sum_{i=1}^{n} Y_{i}(x)\right)= & H(x)(1-H(x)) \\
& +2 \sum_{i=1}^{\infty}\left[\widetilde{\mathrm{P}}\left(\left\{Z_{1} \leq x, Z_{i+1} \leq x\right\}\right)-H(x)^{2}\right] .
\end{aligned}
$$

Moreover, we may apply Theorem 1.2 of [29] to $\operatorname{var}\left(\sum_{i=1}^{n} Y_{i}(x)\right)$ to obtain

$$
\frac{1}{n} \operatorname{var}\left(\sum_{i=1}^{n} Y_{i}(x)\right) \leq 4 \int_{0}^{1} \alpha^{-1}\left(\frac{u}{2}\right) Q(u)^{2} \mathrm{~d} u,
$$

where $Q(u):=\sup \left\{y \in \mathbb{R} \mid \widetilde{\mathrm{P}}\left(\left\{\left|Y_{1}(x)\right|>y\right\}\right)>u\right\}$ and $\alpha^{-1}(u / 2):=\sup \{i \in \mathbb{N} \mid \alpha(i)>$ $u / 2\}(\sup \varnothing:=0)$.

It is easy to check that $Q(u)=1$ if $H(x)>u$ and $Q(u)=0$ otherwise. Moreover, by the assumption on $(\alpha(i))_{i \in \mathbb{N}}$, we obtain $\alpha^{-1}(u / 2) \leq\left[\ln \left(2 \bar{\alpha}_{0}\right)-\ln (u)\right] / \bar{\alpha}_{1}$. Thus,

$$
\frac{1}{n} \operatorname{var}\left(\sum_{i=1}^{n} Y_{i}(x)\right) \leq 4 \int_{0}^{H(x)} \frac{\ln \left(2 \bar{\alpha}_{0}\right)-\ln (u)}{\bar{\alpha}_{1}} \mathrm{~d} u=4 \frac{H(x)\left[1-\ln \left(H(x) /\left(2 \bar{\alpha}_{0}\right)\right)\right]}{\bar{\alpha}_{1}} .
$$

Using an analogous line of reasoning, an additional application of Theorem 1.2 of [29] to $\operatorname{var}\left(\sum_{i=1}^{n}\left[1-Y_{i}(x)\right]\right)$ leads to

$$
\begin{aligned}
\frac{1}{n} \operatorname{var}\left(\sum_{i=1}^{n} Y_{i}(x)\right) & \leq 4 \int_{0}^{1-H(x)} \frac{\ln \left(2 \bar{\alpha}_{0}\right)-\ln (u)}{\bar{\alpha}_{1}} \mathrm{~d} u \\
& =4 \frac{[1-H(x)]\left[1-\ln \left((1-H(x)) /\left(2 \bar{\alpha}_{0}\right)\right)\right]}{\bar{\alpha}_{1}} .
\end{aligned}
$$

Since $\lim _{\gamma \rightarrow 0_{+}} \exp ([\gamma-1] / \gamma]=0$, we can find some $\gamma \in(0, \eta)$ such that $t_{\gamma}:=2 \bar{\alpha}_{0} \exp ([\gamma-$ $1] / \gamma) \in(0,1)$. Then routine considerations yield

$$
\max _{t \in(0,1)} t^{\gamma}\left[1-\ln \left(\frac{t}{2 \bar{\alpha}_{0}}\right)\right]=t_{\gamma}^{\gamma}\left[1-\ln \left(\frac{t_{\gamma}}{2 \bar{\alpha}_{0}}\right)\right]=\frac{\left(2 \bar{\alpha}_{0}\right)^{\gamma} \exp (\gamma-1)}{\gamma} \leq \frac{2 \bar{\alpha}_{0} \exp (\gamma-1)}{\gamma} \text {. }
$$

Hence, by (C.2) and (C.3),

$$
\begin{aligned}
\frac{1}{n} \operatorname{var}\left(\sum_{i=1}^{n} Y_{i}(x)\right) & \leq \frac{8 \bar{\alpha}_{0} \exp (\gamma-1)}{\gamma}\left(H(x)^{1-\gamma} \wedge(1-H(x))^{1-\gamma}\right) \\
& \leq \frac{8 \bar{\alpha}_{0} \exp (\gamma-1)}{\gamma} 2^{1-\gamma}[H(x)(1-H(x))]^{1-\gamma} \\
& \leq \frac{16 \bar{\alpha}_{0}}{\gamma}[H(x)(1-H(x))]^{1-\eta} .
\end{aligned}
$$

Then in view of (C.1) we conclude that

$$
H(x)(1-H(x))+2 \sum_{i=1}^{\infty}\left[\widetilde{\mathrm{P}}\left(\left\{Z_{1} \leq x, Z_{i+1} \leq x\right\}\right)-H(x)^{2}\right] \leq \frac{16 \bar{\alpha}_{0}}{\gamma}[H(x)(1-H(x))]^{1-\eta}
$$

for every $x \in \mathbb{R}$, which completes the proof. 


\section{Acknowledgement}

The authors would like to thank the anonymous referee for a careful reading.

\section{References}

[1] Artzner, P., Delbaen, F., Eber, J.-M. and Heath D. (1999). Coherent measures of risk. Math. Finance 9, 203-228.

[2] Athreya, K. B. And Lahiri, S. N. (2006). Measure Theory and Probability Theory. Springer, New York.

[3] BäUerle, N. AND Müller, A. (2006). Stochastic orders and risk measures: consistency and bounds. Insurance Math. Econom. 38, 132-148.

[4] Bellini, F. and Rosazza Gianin, E. (2008). On Haezendonck risk measures. J. Banking Finance 32, $986-994$.

[5] Ben Hariz, S. (2005). Uniform CLT for empirical process. Stoch Process. Appl. 115, 339-358.

[6] Beutner, E. And Zähle, H. (2010). A modified functional delta method and its application to the estimation of risk functionals. J. Multivariate Anal. 101, 2452-2463.

[7] Billingsley, P. (1968). Convergence of Probability Measures. John Wiley, New York.

[8] Denneberg, D. (1994). Non-Additive Measure and Integral. Kluwer, Dordrecht.

[9] Edgar, G. A. And Sucheston, L. (1992). Stopping Times and Directed Processes. Cambridge University Press.

[10] FISCHER, T. (2003). Risk allocation by coherent risk measures based on one-side moments. Insurance Math. Econom. 32, 135-146.

[11] Föllmer, H. AND Schied, A. (2011). Stochastic Finance, 3rd edn. Walter de Gruyter, Berlin.

[12] Goovaerts, M. J., KaAs, R., Dhaene, J. And Tang, Q. (2004). Some new classes of consistent risk measures. Insurance Math. Econom. 34, 505-516.

[13] Jouini, E., Schachermayer, W. And Touzi, N. (2006). Law invariant risk measures have the Fatou property. Adv. Math. Econom. 9, 49-71.

[14] Kaas, R., Goovaerts, M., Dhaene, J. and Denuit, M. (2008). Modern Actuarial Risk Theory, 2nd edn. Springer, Berlin.

[15] KRÄTSCHMER, V. (2007). On $\sigma$-additive robust representation of convex risk measures for unbounded financial positions in the presence of uncertainty about the market model. SFB 649 discussion paper 2007-010. Available at http://sfb649.wiwi.hu-berlin.de/papers/pdf/SFB649DP2007-010.pdf.

[16] KrätSCHMER, V. AND ZäHLE, H. (2011). Sensitivity of risk measures with respect to the normal approximation of total claim distributions. Insurance Math. Econom. 49, 335-344.

[17] Lifshits, M. A. (1982). On the absolute continuity of distributions of functionals of random processes. Theory Prob. Appl. 27, 600-607.

[18] LindnER, A. M. (2008). Stationarity, mixing, distributional properties and moments of GARCH $(p, q)$ processes. In Handbook of Financial Time Series, eds T. G. Andersen et al. Springer, Berlin, pp. 43-69.

[19] Masuda, H. (2007). Ergodicity and exponential $\beta$-mixing bounds for multidimensional diffusions with jumps. Stoch. Process. Appl. 117, 35-56.

[20] McNeIl, A., Frey, R. and Embrechts, P. (2005). Quantitative Risk Management. Princeton University Press.

[21] Moknadem, A. (1988). Mixing properties of ARMA processes. Stoch. Process. Appl. 29, 309-315.

[22] Müller, A. (2010). Expectiles as risk measures. Preprint, University of Siegen.

[23] Müller, A. And Stoyan, D. (2002). Comparison Methods for Stochastic Models and Risks. John Wiley, Chichester.

[24] Newey, W. K. And Powell, J. L. (1987). Asymmetric least squares estimation and testing. Econometrica 55, 819-847.

[25] Pflug, G. and Wozabal, N. (2010). Asymptotic distribution of law-invariant risk functionals. Finance Stoch. 14, 397-418.

[26] Puri, M. L. and Tran, L. T. (1980), Empirical distribution functions and functions of order statistics for mixing random variables. J. Multivariate Anal. 10, 405-425.

[27] Rajput, R. S. (1972). Gaussian measures on $L_{p}$-spaces, $1 \leq p<\infty$. J. Multivariate Anal. 2, $382-403$.

[28] Römisch, W. (2006). Delta method, infinite dimensional. In Encyclopedia of Statistical Sciences, 2nd edn, eds S. Kotz et al., John Wiley, New York.

[29] Rio, E. (1993). Covariance inequalities for strongly mixing processes. Ann. Inst. H. Poincaré Prob. Statist. 29, 587-597.

[30] Ruszczynski, A. And Shapiro, A. (2006). Optimization of convex risk functions. Math. Operat. Res. 31, 433-452.

[31] Shorack, G. R. (2000). Probability for Statisticians. Springer, New York.

[32] VAn der VAart, A. W. (1998). Asymptotic Statistics. Cambridge University Press. 